\title{
Remarks on the Abraham-Minkowski problem, from the formal and from the experimental side
}

\author{
Iver Brevik ${ }^{1}$, Masud Chaichian², and Ion I. Cotăescu ${ }^{3}$ \\ ${ }^{1}$ Department of Energy and Process Engineering, \\ Norwegian University of Science and Technology, N-7491 Trondheim, Norway \\ ${ }^{2}$ University of Helsinki and Helsinki Institute of Physics, FIN-00014 Helsinki, Finland and \\ 3 West University of Timisoara, V. Parvan Ave. 4, 300223 Timisoara, Romania
}

(Dated: November 16, 2020)

\begin{abstract}
We analyze the Abraham-Minkowski problem known from classical electrodynamics from two different perspectives. First, we follow a formal approach, implying use of manifolds with curved space sections in accordance with Fermat's principle, emphasizing that the resulting covariant and contravariant components of the photon four-momentum is a property linked to the Minkowski theory only. There is thus no link to the Abraham theory in that context. Next we turn to the experimental side, giving a brief account of older and newer radiation pressure experiments that clearly show how the Minkowski photon momentum is preferable under optical conditions. Under low-frequency conditions, where experimental detection of the individual oscillations predicted by the Abraham term are possible, the picture is however quite different. PACS: 04.20.Cv
\end{abstract}




\section{INTRODUCTION}

A historical controversy concerns the Abraham [1], versus the Minkowski [2], definitions of the momentum of a photon propagating in a continuous medium (some references are [3-12]). In classical electrodynamics the Minkowski momentum density is $\mathbf{g}^{\mathrm{M}}=\mathbf{D} \times \mathbf{B}$, whereas the Abraham momentum density is $\mathbf{g}^{\mathrm{A}}=\mathbf{E} \times \mathbf{H} / c^{2}$. The Planck principle of inertia of energy, saying that the relationship between momentum density $\mathbf{g}$ and the Poynting vector $\mathbf{S}$ is $\mathbf{g}=\mathbf{S} / c^{2}$, is thus broken in the Minkowski case.

The phenomenological theory can be quantized if the underlying electromagnetic energymomentum tensor is such that it permits the construction of the Fock space. In practice, this will be possible only for the Minkowski case, since then the energy-momentum tensor is divergence-free, thus securing four-vector properties of the energy and momentum components of the radiation field (cf., for instance, Refs. [13] and [14].

The Abraham-Minkowski problem has many facets, and we will here focus on two of them. First we will approach the problem from a formal side, related to the wave-particle duality. As formulated by the Barnett [15], the Abraham and Minkowski momenta are respectively the kinetic and canonical optical momenta. The formalism from general relativity can be used to illustrate these formal properties, by considering the optical medium as a pseudoRiemannian manifold where the photon momenta satisfy the standard null condition. We assume for simplicity a transparent and isotropic medium, at rest only. Thus, the photon momenta $\mathbf{p}^{\mathrm{A}}$ and $\mathbf{p}^{\mathrm{M}}$ are related via

$$
E=\left|\mathbf{p}^{\mathrm{A}}\right| n c=\left|\mathbf{p}^{\mathrm{M}}\right| \frac{c}{n}
$$

where $E$ is the photon energy.

Here we should emphasize that these formal properties do not reflect the physical difference between the Minkowski and Abraham theories. The quite elegant formalism relates to the four-vector property of photon momentum, a property possessed by the Minkowski theory but not by the Abraham one. This point has some times led to misunderstandings.

We will in the next item 2 consider photons propagating in a $(1+3)$-dimensional localMinkowskian curved manifold $M$ in which the propagation occurs along null geodesics while the electromagnetic waves are determined by the Maxwell equations without sources. Omitting gravity, these requirements are satisfied by a pseudo-Riemannian metric (a Fermat 
manifold) resulting from the invariant Fermat principle applied to the proper time instead of the optical length as in the general relativistic case [16-20].

We then move on to physical considerations, presenting a brief review over optical experiments and singling out those that actually show the importance of the Minkowski photon momentum and those that do not. Actually, most of the experiments are not important in this respect, as they show only the action of electromagnetic forces at dielectric boundaries. Forces of that type are equally well described by the Abraham tensor as by the Minkowski tensor, and have little to do with photon momentum in matter.

Finally, as a contrast, we focus attention on how the theory becomes modified if the frequencies are so low that the fluctuations arising from the so-called Abraham term become detectable directly.

\section{FERMAT MANIFOLDS}

Let $M$ denote a transparent isotropic ideal static medium at rest in which the light is neither absorbed nor dispersed. We consider the static metric $g$ of components $g_{00}=$ $-1, \quad g_{i j}(x)=\delta_{i j} n(x)^{2}, \quad i, j, k, \ldots=1,2,3$ where $n(x)$ is the refractive index. Introduce Cartesian coordinates, $x^{\mu}$, and write the line element as

$$
d s^{2}=g_{\mu \nu}(x) d x^{\mu} d x^{\nu}=-c^{2} d t^{2}+n(x)^{2}\left[\left(d x^{1}\right)^{2}+\left(d x^{2}\right)^{2}+\left(d x^{3}\right)^{2}\right],
$$

where $n(x)$ depends only on the space coordinates $x^{i}$.

In this geometry test particles move along geodesics $x^{i}=x^{i}(t)$, with velocity $\dot{x}^{i}=\frac{d x^{i}}{d t}=v^{i}$, determined from the geometric variational principle

$$
\delta S=\delta \int_{t_{1}}^{t_{2}} \sqrt{-d s^{2}}=\delta \int_{t_{1}}^{t_{2}} \sqrt{c^{2}-n^{2}\left[\left(\dot{x}^{1}\right)^{2}+\left(\dot{x}^{2}\right)^{2}+\left(\dot{x}^{3}\right)^{2}\right]} d t=0 .
$$

This can be looked upon as the relativistically invariant Fermat's principle, applied to proper time instead of to optical path [20]. The photons propagate in $M$ along null geodesics whose equations of motion are

$$
\dot{v}^{k}=\vec{v}^{2} \frac{1}{n} \partial_{k} n-2 \frac{v^{k}}{n}\left(v^{i} \partial_{i} n\right), \quad k=1,2,3
$$

allow a prime integral that can be set as $n^{2} \vec{v}^{2}=c^{2}$ corresponding to the null condition $d s^{2}=0$. Thus we recognize the simple relation $v=\frac{c}{n}$ that holds for isotropic media. 
Now, we introduce the dual (or contravariant) metric tensor $\bar{g}$, with components

$$
g^{\mu \nu}=\operatorname{diag}\left(-1, \frac{1}{n^{2}}, \frac{1}{n^{2}}, \frac{1}{n^{2}}\right) .
$$

They appear in the Maxwell equations $\nabla_{\mu} F^{\mu \nu}=0$, with $g=|\operatorname{det}(g)|$. The Lorentz condition is $\nabla_{\mu} A^{\mu}=0$. Combining these equations one obtains the wave equation for the electromagnetic potential $A_{\mu}$.

On the manifold $M$ we may introduce orthogonal non-holonomic local frames defined by the tetrad fields $e_{\hat{\alpha}}=e_{\hat{\alpha}}^{\mu} \partial_{\mu}$ and the corresponding co-frames given by the 1 -forms $\omega^{\hat{\alpha}}=$ $\omega_{\mu}^{\hat{\alpha}} d x^{\mu}$, labeled by local indices $\hat{\alpha}, \ldots \hat{\mu}, \hat{\nu} \ldots=0,1,2,3$. These frames are orthogonal and dual to each other satisfying the duality condition $\omega^{\hat{\alpha}}\left(e_{\hat{\beta}}\right)=\delta_{\hat{\beta}}^{\hat{\alpha}}$ and the orthogonality condition $g\left(e_{\hat{\alpha}}, e_{\hat{\beta}}\right)=\eta_{\hat{\alpha} \hat{\beta}}$. It is convenient to choose the diagonal tetrad gauge,

$$
\begin{array}{cc}
e_{\hat{0}}=\partial_{t}, & e_{\hat{i}}=\frac{1}{n} \partial_{i}, \\
\omega^{\hat{0}}=c d t, & \omega^{\hat{i}}=n d x^{i},
\end{array}
$$

since in this gauge the space axes of the local frame are parallel with those of the space coordinates. However, the normalization is different such that the line element (2) can be written as $d s^{2}=\eta_{\hat{\alpha} \hat{\beta}} \omega^{\hat{\alpha}} \omega^{\hat{\beta}}$ where $\eta=\operatorname{diag}(-1,1,1,1)$ is the Minkowski metric of the flat model of $M$. In our convention the natural indices are raised or lowered by the metric tensor $g$ while for the local indices we have to use the metric $\eta$.

On the photon four-momentum.- Photon momentum is defined by the de Broglie relations that hold in static and homogeneous media where the wave vector is well-defined. For a homogeneous medium $n$ is independent of the space coordinates and so the Fermat manifold remains flat. In such a manifold the photon moves along linear null geodesics with constant velocity satisfying the null condition. In the Coulomb gauge, with $A_{0}=0$, the wave equation becomes

$$
\square A_{i}=\Delta A_{i}-\frac{n^{2}}{c^{2}} \partial_{t}^{2} A_{i}=0,
$$

corresponding to the speed $c^{\prime}=c / n<c$ in $M$.

The wave equation has plane-wave solutions of the form $A_{i}(t, \vec{x}) \propto \varepsilon_{i} e^{i k_{\mu} x^{\mu}}$, where $\varepsilon$ is the polarization vector while $k_{\mu}$ is the wave four-vector. Then, according to the de Broglie formulas, we may define the covariant components $p_{\mu}$ of the photon four-vector,

$$
p_{\mu}=\hbar k_{\mu}=\left(-\frac{E}{c}, p_{1}, p_{2}, p_{3}\right) \text {. }
$$


Writing the null condition in the form

$$
0=g^{\mu \nu} p_{\mu} p_{\nu}=\frac{1}{n^{2}} p_{i} p_{i}-\frac{E^{2}}{c^{2}}
$$

it is natural to identify $p_{i}$ with the spatial components of the Minkowski photon fourmomentum, $p^{\mathrm{M}} \equiv\left|\mathbf{p}^{\mathrm{M}}\right|=n E / c$. It agrees with the second member of Eq. (1).

We may define also the contravariant components of the four-momentum,

$$
p^{\mu}=g^{\mu \nu} p_{\mu}=\left(\frac{E}{c}, \frac{p_{1}}{n^{2}}, \frac{p_{2}}{n^{2}}, \frac{p_{3}}{n^{2}}\right)=\left(\frac{E}{c}, p^{1}, p^{2}, p^{3}\right)
$$

and write the null condition in the form

$$
0=g_{\mu \nu} p^{\mu} p^{\nu}=n^{2} p^{i} p^{i}-\frac{E^{2}}{c^{2}}
$$

In addition, thanks to our approach we can define the local momentum $\hat{p}$ in the orthogonal frames defined by the tetrad gauge (6) and (17). According to the general definitions, $\hat{p}^{\hat{\alpha}}=$ $\omega_{\mu}^{\hat{\alpha}} p^{\mu}$ and $\hat{p}_{\hat{\alpha}}=e_{\hat{\alpha}}^{\mu} p_{\mu}$, we find the components

$$
\hat{p}^{0}=-\hat{p}_{0}=\frac{E}{c}, \quad \hat{p}^{i}=\hat{p}_{i}=n p^{i}=\frac{p_{i}}{n},
$$

which satisfy the identity

$$
0=\eta_{\hat{\alpha} \hat{\beta}} \hat{p}^{\hat{\alpha}} p^{\hat{\beta}}=\left(\hat{p}^{1}\right)^{2}+\left(\hat{p}^{2}\right)^{2}+\left(\hat{p}^{3}\right)^{2}-\frac{E^{2}}{c^{2}} .
$$

The conclusion is that in our manifold $M$ the Abraham-type momentum is the vector field

$$
p_{A}=p^{\mu} \partial_{\mu}=\hat{p}^{\hat{\alpha}} e_{\hat{\alpha}} \in T M,
$$

while that of Minkowski-type the dual co-vector (or 1-form)

$$
p_{M}=p_{\mu} d x^{\mu}=\hat{p}_{\hat{\alpha}} \omega^{\hat{\alpha}} \in T^{*} M
$$

The notations $T M$ and $T^{*} M$ stand for the tangent and respectively cotangent bundles on $M$ [21]. Now the null conditions (10) and (11) as well as their particular cases (11) are given just by the duality relation

$$
p_{M}\left(p_{A}\right)=g\left(p_{A}, p_{A}\right)=\bar{g}\left(p_{M}, p_{M}\right)=0 .
$$

Thus we defined coherently the momenta $p_{A}$ and $p_{M}$ in flat Fermat manifolds solving at least formally the Abraham-Minkowski dilemma. 
However, this formal conciliation enters in collision with the actual electrodynamics of continuous media which traditionally cannot be decoupled to special relativity where $p_{\mu}$ and $p^{\mu}$ are the covariant and contravariant components of one and the same Minkowski four-vector. Nevertheless, if we accept as an hypothesis a future non-Minkowskian electrodynamics, in which some media have Fermat geometry, then the above presented formalism could explain why in the Abraham formulation the radiation energy and momentum do not make up a four-vector at all in the sense of special relativity. The reason for this is that the Abraham energy-momentum tensor is not divergence-free, even in a homogeneous medium. Note that this was pointed out by Møller a long time ago [22], and has been emphasized by one of us several authors several times [4, 23, 24] also.

\section{ON EXPERIMENTS}

As many nice radiation pressure experiments have been reported in the literature it becomes important to check if one, or more, of the theoretical explanations of them stands out as the most favorable one. And this actually turns out to be the case. As mentioned above, all optical experiments of this sort that we are aware of, become explainable by the Minkowski theory in a simple and straightforward way. A nontrivial point, however, is whether a specific experiment gives information about photon momentum, or if it merely shows the action of electromagnetic forces at dielectric boundaries. One of us gave recently an overview of this sort [23]; we will not repeat that here, but will give a brief summarizing survey and moreover focus on a very new experiment that also seems to be valuable in this context.

We will start with the general expression for the electromagnetic force density $\mathbf{f}$ in an isotropic medium:

$$
\begin{aligned}
\mathbf{f}= & \rho \mathbf{E}+\mathbf{J} \times \mathbf{B}+\frac{1}{2} \varepsilon_{0} \nabla\left[E^{2} \rho_{m} \frac{\partial \varepsilon}{\partial \rho_{m}}\right]+\frac{1}{2} \mu_{0} \nabla\left[H^{2} \rho_{m} \frac{\partial \mu}{\partial \rho_{m}}\right] \\
& -\frac{1}{2} \varepsilon_{0} E^{2} \nabla \varepsilon-\frac{1}{2} \mu_{0} H^{2} \nabla \mu+\frac{n^{2}-1}{c^{2}} \frac{\partial}{\partial t}(\mathbf{E} \times \mathbf{H}) .
\end{aligned}
$$

We assume no charges or currents, $\rho=\mathbf{J}=0$, assume the material to be nonmagnetic, and omit the two electrostriction and magnetostriction terms ( $\rho_{m}$ is the matter density). We write the constitutive relations as $\mathbf{D}=\varepsilon \varepsilon_{0} \mathbf{E}, \mathbf{B}=\mu_{0} \mathbf{H}$, so that $\varepsilon$ becomes nondimensional. 
The basic expression above thus reduces to the form

$$
\mathbf{f}^{\mathrm{A}}=-\frac{1}{2} \varepsilon_{0} E^{2} \nabla n^{2}+\frac{n^{2}-1}{c^{2}} \frac{\partial}{\partial t}(\mathbf{E} \times \mathbf{H}),
$$

with $n^{2}=\varepsilon$. This is the Abraham force density. The force consists of two terms, the first acting at dielectric boundaries typically, the second (usually called the Abraham term) acting in the bulk. The second term is however rapidly fluctuating out in an optical wave.

The Minkowski force density $\mathbf{f}^{\mathrm{M}}$ is equal to the expression above, except from the Abraham term,

$$
\mathbf{f}^{\mathrm{M}}=-\frac{1}{2} \varepsilon_{0} E^{2} \nabla n^{2}
$$

In a homogeneous interior this force thus vanishes.

The action of the Abraham term is to produce an accompanying mechanical momentum density

$$
\mathbf{g}_{\text {mech }}=\frac{n^{2}-1}{c^{2}} \mathbf{E} \times \mathbf{H},
$$

which together with the Abraham momentum density $\mathbf{g}^{\mathrm{A}}=(\mathbf{E} \times \mathbf{H}) / c^{2}$ gives rise to the total propagating Minkowski momentum density

$$
\mathrm{g}^{\mathrm{M}}=\mathrm{g}^{\mathrm{A}}+\mathrm{g}_{\text {mech }}=\mathbf{D} \times \mathbf{B} .
$$

When considering some concrete radiation pressure experiments, the central question becomes in each case: does the observed result show the action of the Abraham force (19), or does it only show the force at the boundaries, i.e., the Minkowski force (201)? We first list a few examples belonging to the same (actually the second) category:

A classic experiment is that of Ashkin and Dziedzic [25], in which a pulsed laser beam at $0.53 \mu \mathrm{m}$ was sent down on a water surface, resulting in a weak outward bulge $(0.9 \mu \mathrm{m}$ height) of the surface. The reason for the weakness was the high surface tension for water. Later experiments, operating with micellar liquids in the vicinity of the critical point, led to much larger surface elevations, about $70 \mu \mathrm{m}$ [26], because of the very significant reduction of surface tension in this case. The recent experiment of Astrath et al. [27] is essentially of Ashkin-Dziedzic type. Experiments on curved surfaces have also been done, by Zhang and Chang on water droplets [28], and by Zhang et al. on mineral oil and water surfaces [29]. An interesting recent experiment of Kundu et al. [30] demonstrates how a horizontal graphene oxide plate becomes deflected when illuminated by a weak continuous laser beam from above. 
All these experiments have one property in common: they are explainable in terms of radiation surface forces on boundaries only (although it is sometimes claimed that they favor the Abraham theory; cf., for instance, the critical note by one of us [24] on this point). They thus show the action of the force (20). In that way they do not have a direct bearing on the Abraham-Minkowski problem.

One may ask: which experiments are then able to show the action of the Abraham term? We may summarize a few cases of that kind as follows:

\section{Radiation pressure on a metallic suspended mirror}

The classic experiments of Jones et al. [31, 32], measuring the pressure on a metallic mirror immersed in a dielectric liquid, still play a prominent role. The pressure was found to be proportional to the dielectric constant $n$ of the liquid, thus supporting the reality of the flowing Minkowski momentum density $\mathbf{g}^{\mathrm{M}}$.

\section{Photon drag in semiconductors}

The semiconductor experiment of Gibson et al [33] is also important in our context. The longitudinal electric field produced by the momentum transfer from an incident radiation field was measured. This field was produced by charges driven down in the dielectric rod. The agreement with the Minkowski prediction for photon momentum was quite good (this experiment was also commented upon in Ref. [34]).

\section{Direct measurement of photon momentum}

A third and noteworthy experiment is that of Campbell et al. [35], making a direct measurement of the photon recoil in a Bose-Einstein condensate. The photon momentum was found to be $\hbar k=\hbar n \omega / c$, just as predicted in the Minkowski theory.

\section{Photon drag in thin metal films}

An interesting and very recent result is the measurement of Minkowski's photon momentum, actually as a by-product, in the photon drag experiment of Strait et al. [36]. The main purpose of this experiment was not to test Minkowski momentum but rather to focus attention on the possibility of a counter intuitive reversion of the direction of the induced electron flow caused by the photons. As this experiments is apparently not well known in the radiation pressure community, we will go into some detail, focusing on the part of the experiment on main importance here. 
The setup was a $\mathrm{SiO}_{2}$ hemisphere resting on a thin, flat $\mathrm{Au}$ layer. Incident photons coming from the ambient air region, inclined at an angle $\theta$ with the vertical, propagated orthogonally through the hemispherical surface and exerted subsequently a drag on the electrons in the plate. The electromagnetic energy reflected back from the spherical surface was low and neglected to the actual order of accuracy. We will consider only the case of $s$ polarization, for which the radiation force was found to act in the forward direction, what is one would intuitively expect. The thickness of the Au plate was $35 \mathrm{~nm}$, the free electron density was $5.9 \times 10^{22} \mathrm{~cm}^{-3}$, and an optical pulse train of wavelength $800 \mathrm{~nm}$ was used. The angle $\theta$ varied between 0 and $\pm 60^{\circ}$.

The same measurement was made in the absence of the semicylinder. Comparison of the in-plane transduction factors denoted by $\xi_{g}^{s}$ (glass-metal) and $\xi_{f}^{s}$ (free space) showed that to their ratio was equal to 1.53. This is roughly the same as the refractive index 1.45 for $\mathrm{SiO}_{2}$. As the experimenters concluded themselves, this strongly indicates that the $s$ wave photonic voltage was proportional to the Minkowski momentum $n \hbar \omega / c$. The experiment is clearly related to the photon drag experiment discussed above.

The case of $p$ polarization was more complicated, and might involve second order modification channels as discussed in Ref. [36]. Analog results to those above were then not found.

\section{The case of low frequencies}

Thus far, we have considered optical frequencies and the obvious usefulness of Minkowski's momentum under those circumstances. Quite a different picture arises if we turn to the case of low frequencies, for which the oscillations predicted by the Abraham force term in Eq. (19) become directly observable. The prominent example of this kind of experiment is that of the Walkers and Lahoz reported in Refs. [37] and [38]. A dielectric high-permittivity disk (barium titanate, $\varepsilon=3620$ was suspended in the gravitational field and acted as a torsional pendulum. The inner and outer disk surfaces were coated with aluminium, and a harmonic voltage of magnitude $V_{0} \approx 260 \mathrm{~V}$ of the same periodicity as the mechanical pendulum was applied across the surfaces. When a constant axial magnetic field was applied, there arouse an axial Abraham torque $N_{z}$ on the shell. With $\omega / 2 \pi \approx 0.4 \mathrm{~Hz}$, the predicted torque of magnitude $N_{z} \approx 4 \times 10^{-10} \mathrm{Nm}$ was found to agree with the observations to within $\pm 10 \%$. This is a clear demonstration of the reality of the Abraham force, under these special 
conditions. We have actually emphasized the importance of this experiment before [4], but it still does not seem to be well known.

\section{CONCLUDING REMARKS}

We have shed light on the Abraham-Minkowski problem from two quite different perspectives. Our formal approach was related to the Gordon metric $g_{G}$ which is also a Finslerian

metric [39], defined in classical relativistic electrodynamics [40]. If the medium is isotropic and at rest, the Fermat and Gordon metrics are related through the conformal transformation, $g=n^{2} g_{G}$. It means that these are geometrically non-equivalent even though they may produce similar null geodesics.

It is however to be borne in mind that these formal developments, although instructive, are not able to solve the physical Abraham-Minkowski problem. For that purpose, one should look at the experiments. We have listed a few experiments that we consider to be critical enough to test the Minkowski photon momentum. As mentioned, we emphasize that all experiments that we are aware of in optics, are most conveniently explained in terms of the Minkowski theory.

\section{Acknowledgments}

We are very grateful to Merab Gogberashivili, Friedrich Hehl and Yuri Obukhov for several useful discussions at different stages of this work, and to Jared H. Strait for correspondence concerning Ref. [36].

[1] M. Abraham, On the electrodynamics of moving bodies, Rend. Circ. Mat. Palermo 28, 1-28 (1909).

[2] H. Minkowski, Die Grundgleichungen für die elektromagnetischen Vorgn̈ge in bewegten Körpern, Nachr. Ges. Wiss. Göttingen (1908) 53.

[3] U. Leonhardt, Optics - Momentum in an uncertain light, Nature (London) 444 (2006) 823.

[4] I. Brevik, Experiments in phenomenological electrodynamics and the electromagnetic energymomentum tensor, Phys. Rep. 52 (1979) 133. 
[5] R. N. C. Pfeifer, T. A. Nieminen, N. R. Heckenberg, and H. Rubinsztein-Dunlop, Momentum of an electromagnetic wave in dielectric media, Rev. Mod. Phys. 79 (2007) 1197.

[6] N. L. Balazs, The energy-momentum tensor of the electromagnetic field inside matter, Phys. Rev. 91 (1953) 408.

[7] P. W. Milonni and R. W. Boyd, Recoil and photon momentum in a dielectric, Laser Phys. 15 (2005) 1.

[8] D. H. Bradshaw, Z. Shi, R.W. Boyd, and P.W. Milonni, Electromagnetic momenta and forces in dispersive dielectric media, Opt. Commun. 283 (2010) 650; arXiv: 0910.0770.

[9] M. J. Padgett, On diffraction within a dielectric medium as an example of the Minkowski formulation of optical momentum, Opt. Express 16 (2008) 20864.

[10] I. Brevik, Phenomenological photons and the uncertainty principle, Eur. J. Phys. 2 (1981) 37.

[11] Yu. N. Obukhov, Electromagnetic energy and momentum in moving media, Ann. Phys. (Berlin) 17 (2008) 830; arXiv: 0808.1967v1.

[12] T. Ramos, G. F. Rubilar and Y. N. Obukhov, First principles approach to the AbrahamMinkowski controversy for the momentum of light in general linear non-dispersive media, J. Opt. 17 (2015) 025661; arXiv: 1310.0518.

[13] I. Brevik, Minkowski momentum resulting from a vacuum-medium mapping procedure, and a brief review of Minkowski momentum experiments, Annals of Physics 377 (2017) 10.

[14] I. Brevik, Classical and quantal aspects of Minkowski's four-momentum in analog gravity, Phys. Rev. A (2020).

[15] S. M. Barnett, Resolution of the Abraham-Minkowski Dilemma, Phys. Rev. Letters, 104 (2010) 070401.

[16] T. Ramos, G.F. Rubilar, and Yu.N. Obukhov, Relativistic analysis of the dielectric Einstein box: Abraham, Minkowski and total energy-momentum tensors, Phys. Lett. A 375 (2011) 1703; arXiv:1103.1654v2.

[17] H. Weyl, The theory of gravitation, Annalen der Physik 54 (1917) 117.

[18] W. Pauli, Theory of Relativity (Pergamon Press, Oxford,England 1958).

[19] C. W. Misner, K. S. Thorne, and J. A. Wheeler, Gravitation (Freemann, San Francisco 1973)

[20] L. D. Landau and E. M. Lifshitz, The Classical Theory of Fields (Pergamon Press, Oxford, England 1975)

[21] J. M. Lee, Manifolds and Differential Geometry (Texas Tech. University, Lubbock, 2009). 
[22] C. Møller, The Theory of Relativity, 2nd ed. (Clarendon Press, 1972).

[23] I. Brevik, Radiation forces and the Abraham-Minkowski problem, Mod. Phys. Lett. A 33 (2018) 1830006.

[24] I. Brevik, Analysis of recent interpretations of the Abraham-Minkowski problem, Phys. Rev. A 98 (2018) 043847.

[25] A. Ashkin and J. M. Dziedzic, Radiation pressure on a free liquid surface, Phys. Rev. Lett. 30 (1973) 19.

[26] A. Casner and J. P. Delville, Laser-induced hydrodynamic instability of fluid interfacs, Phys. Rev. Lett. 90 (2003) 144503.

[27] N. G. Astrath, L. C. Malacarne, M. L. Baesso, G. V. Lukasievicz, and S. E. Bialkowski, Unravelling the effects of radiation forces in water, Nature Commun. 5 (2014) 4363.

[28] J. Z. Zhang and R. K. Chang, Shape distortion of a single water droplet by laser-induced electrostriction, Opt. Lett. 13 (1988) 916.

[29] L. Zhang, W. She, N. Peng, an U. Leonhardt, Experimental evidence for Abraham pressure of light, New J. Phys. 17 (2015) 053035.

[30] A. Kundu, R. Rani, and K. S. Hazra, Graphene oxide demonstrates experimental confirmation of Abraham pressure on solid surface, Sci. Rep. 7 (2017) 42538.

[31] R. V. Jones and J. C. Richards, The pressure of radiation in a refractive medium, Proc. Roy. Soc. (London) A 221 (1954) 480.

[32] R. V. Jones and B. Leslie, The measurement of optical radiation pressure in dispersive media, Proc. Roy. Soc. (London) A 360 (1978) 347.

[33] A. F. Gibson, M. F. Kimmitt, A. O. Koohian, D. E. Evans, and G. F. D. Levy, A study of radiation pressure in a refractive medium by the photon drag effect, Proc. Roy. Soc. (London) A 370 (1980) 303.

[34] I. Brevik, Photon drag experiment and the electromagnetic momentum in matter, Phys. Rev. B 33 (1986) 1058.

[35] G. K. Campbell, A. E. Leanhardt, J. Mun, M. Boyd, E. W. Streed, W. Ketterle, and D. E. Pritchard, Photon recoil momentum in dispersive media, Phys. Rev. Lett. 94 (2005) 170403.

[36] J. H. Strait, G. Holland, W. Zhu, C. Zhang, B. R. Ilic, A. Agrawal, D. Pacifici, and H. J. Lezec, Revisiting the photon-drag effect in metal films, Phys. Rev. Lett. 123 (2019) 05390

[37] G. B. Walker, D. G. Lahoz and G. Walker, Measurement of Abraham force in a barium-titanate 
specimen, Can. J. Phys. 53, 2577 (1975).

[38] G. B. Walker and D. G. Lahoz, Experimental observation of Abraham force in a dielectric, Nature (London) 253, 339 (1975).

[39] W. Gordon, Light propagation according to the relativity theory, Annals of Physics (New York), 22 (1923) 421.

[40] R. T. Thompson, Covariant electrodynamics in linear media: Optical metric, Phys. Rev. D 97 (2018) 065001. 